\title{
The role of meaning in visual working memory: Real- world objects, but not simple features, benefit from deeper processing
}

\author{
Timothy F. Brady \\ Department of Psychology \\ University of California, San \\ Diego
}

\author{
Viola S. Störmer \\ Department of Psychological and Brain \\ Sciences, Dartmouth College
}

\footnotetext{
Please address correspondence to:

Timothy Brady

9500 Gilman Dr. \#0109

McGill Hall 5322

La Jolla, CA, 92093

timbrady@ucsd.edu
}

Word count: 9826 


\begin{abstract}
Visual working memory is a capacity-limited cognitive system used to actively store and manipulate visual information. Visual working memory capacity is not fixed, but varies by stimulus type: stimuli that are more meaningful are better remembered. In the current work, we investigate what conditions lead to the strongest benefits for meaningful stimuli. We propose that in some situations, participants may be prone to try to encode the entire display holistically (i.e., in a quick 'snapshot'), encouraging participants to treat objects simply as meaningless colored 'blobs', rather than processing them individually and in a high-level way, which could reduce benefits for meaningful stimuli. In a series of experiments we directly test whether realworld objects, colors, perceptually-matched less-meaningful objects, and fully scrambled objects benefit from deeper processing. We systematically vary the presentation format of stimuli at encoding to be either simultaneous - encouraging a parallel, 'take-a-quick-snapshot' strategy - or present the stimuli sequentially, promoting a serial, each-item-at-once strategy. We find large advantages for meaningful objects in all conditions, but find that real-world objects - and to a lesser degree lightly scrambled, still meaningful versions of the objects - benefit from the sequential encoding and thus deeper, focused-on-individual-items processing, while colors do not. Our results suggest single feature objects may be an outlier in their affordance of parallel, quick processing, and that in more realistic memory situations, visual working memory likely relies upon representations resulting from in-depth processing of objects (e.g., in higher-level visual areas) rather than solely being represented in terms of their low-level features.
\end{abstract}

Keywords: visual working memory, visual long-term memory, memory encoding, depth of processing 


\section{Introduction}

Visual working memory is a capacity-limited cognitive system used to actively store and manipulate visual information (Baddeley, 2012; Cowan, 2001). While theories generally agree that its capacity is limited, they differ in terms of what these limits are and how they arise. Prominent models of working memory have promoted the view of a 'fixed' limit of working memory, arguing that a particular number of objects can be stored at once, regardless of what these objects are (e.g., Luck \& Vogel, 1997; Awh, Barton \& Vogel, 2007), or that a fixed amount of resources can be distributed among the to-be-remembered stimuli (e.g., Bays et al., 2009) within each of a small number of feature dimensions (i.e., color, orientation). Support for these strong fixed capacity models comes from numerous studies examining visual working memory limits using simple stimuli like colored squares, oriented lines or novel shapes (e.g., Zhang \& Luck, 2008); all stimuli about which participants have minimal background knowledge or expectations. These simple, meaningless stimuli are often assumed to best assess the core capacity of working memory because they have no semantic associations and are repeated from trial-to-trial, which minimizes participants' ability to use other memory systems, like episodic visual long-term memory, to support memory performance (Cowan, 2001; Lin \& Luck, 2012).

Using such simple stimuli, past studies have often used short encoding times (generally $<500 \mathrm{~ms}$ ), assuming that working memory fills up quickly, and have argued that the stark limits on performance are truly limits of working memory - that is, the limits on performance do not arise from limited encoding times or limits in perceptual processing (e.g., Luck \& Vogel, 1997; Alvarez \& Cavanagh, 2004; Bays et al. 2011; Tsubomi, Fukuda, Watanabe, \& Vogel, 2013; Vogel et al. 2006). For example, Luck and Vogel (1997) argued the same capacity limits appeared regardless of encoding time, which they said meant such limits arose from "limitations in storage capacity rather than limitations in perceiving or encoding the stimuli"; similarly, Alvarez and Cavanagh (2004) claimed that their results were from a storage limit, not an encoding limit, because "all of the information that can be stored is acquired in less than $500 \mathrm{~ms}$.

In stark contrast to these findings of fixed performance regardless of encoding time in simple stimuli, we recently found working memory performance to be higher for real-world objects than for simple stimuli, particularly when participants were given longer time to encode these items 
(Brady, Störmer, \& Alvarez, 2016). In that study, participants were asked to either remember objects or colors over a short delay, and discriminate one of the remembered stimuli in a 2alternative forced choice against a maximally distinct foil object or color. Specifically at long encoding times (1s and 2s), participants better remembered real-world objects than colors. What drives these differences in capacity? One possible explanation is that working memory operates equally well on both stimulus types, but real-world objects can additionally benefit from the high-capacity episodic long-term memory system or a form of more accessible long-term memories (Cowan, 1988; Quirk et al., 2020). It is sometimes speculated that such additional systems could particularly play a role with long encoding times (e.g., Lin \& Luck, 2012), although there is little direct evidence to suggest this. Notably, claims of long-term memory involvement are not suggesting any use of "long"-term storage: the only way to correctly respond to the test probe in such studies is to pick the item that was seen on that particular trial at that particular location, as both the studied items and foil items are equally familiar real-world objects; thus, information must be used about that specific trial. Instead, such objections are based on the suggestion that people can perform such binding of an object to a location from 1 second ago not only using an online, active maintenance system, but also, in some situations, by using a fundamentally different, offline system, and that the usage of such a system applies only to some stimuli in some situations and it cannot be distinguished behaviorally whether this additional offline system was used.

To directly test this idea, and examine whether the performance benefits for real-world objects at long encoding times were due to the recruitment of non-active memory systems, such as 'long'-term memory, in our previous study we examined the contralateral delay activity (CDA) a neural marker of how much information is stored actively in working memory (Vogel \& Machizawa, 2004). We found, as in many previous CDA studies, that the CDA amplitude tracked behavioral performance increases. This provides evidence that real-world objects were stored actively in visual working memory - just like colors or other basic features. Specifically, we found that in line with behavioral performance, objects showed greater CDA at longer encoding times than shorter encoding times, consistent with additional information being actively held in mind; and that at long encoding the CDA was reliably greater for remembering 5 objects than for remembering 5 colors (but not different when the amount remembered was the same for each stimulus set; e.g., with 3 of each presented). We hypothesized that real-world objects particularly benefited from longer encoding time in our study because this enabled a deeper processing of these stimuli, which may facilitate accessing existing knowledge of these 
stimuli which can be used to help hold them 'online', something that would not be useful for simple stimuli such as colors (Brady et al., 2016).

In particular, people may maintain information in visual working memory not solely in terms of colors and shapes and other 'basic' visual dimensions in low-level visual cortex (e.g., Serences, 2016), but also maintain active representations of the stimuli in higher-level visual regions (e.g., fusiform face area (FFA) for face stimuli: Druzgal \& D'Esposito, 2001; somatosensory regions for hand images: Galvez-Pol et al. 2018), resulting in stronger memories for items that can be meaningfully represented in higher-level brain regions. Consistent with this, Stojanoski et al. (2019) have shown greater ventral stream involvement in visual working memory tasks for meaningful rather than perceptually-matched non-meaningful stimuli. In addition, we have shown that perceptually-matched images that are perceived as a face are not only better remembered in a working memory task than those not perceived as a face, but also elicit a larger CDA (Asp, Störmer \& Brady, 2019), once again showing that 'online' storage in working memory nearly always tracks behavioral performance in these tasks, rather than participants relying on a mix of memory systems only for some stimuli at some encoding times but not others. Consistent with this model of greater engagement of higher-level regions with meaningful stimuli, Salmela et al. (2019) have shown that storing faces in memory results in the storage of both low- and high-level information about them, whereas simple orientation stimuli are stored in a solely low-level way. Furthermore, a significant literature has shown, using behavior alone, that familiarity and knowledge improve performance in short-term memory tasks even with perceptually well-matched or even identical stimuli (e.g., Alvarez \& Cavanagh, 2004; Jackson \& Raymond, 2008; Brady et al. 2009; Curby et al. 2009; Ngiam et al. 2019; O'Donnell, Clement, \& Brockmole, 2018; Sahar et al. 2020; Starr, Srinivasan, Bunge, 2020). For example, familiar faces appear to be easier to remember than unfamiliar faces (Jackson \& Raymond, 2004); and familiar letters, rather than letters from unfamiliar alphabets, are more easily remembered (Ngiam et al. 2019), conceivably due to the ability to recruit high-level features when processing such stimuli.

Several recent studies challenged these previous findings of benefits of knowledge and familiarity for working memory. In particular, two recent studies have challenged the claim of a selective benefit at long encoding times for real-world objects, instead finding benefits from long encoding time for both real-world objects and simple colors (Quirk et al. 2020; Li et al. 2020). These results contest not only the 'higher-capacity for real-world objects' account, but pose a 
serious problem for fixed storage capacity models in general - as they strongly contrast with the standard claim that limits in performance in these paradigms arise primarily from storage limits, not encoding limits (e.g., Luck \& Vogel, 1997; Alvarez \& Cavanagh, 2004; Tsubomi, Fukuda, Watanabe, \& Vogel, 2013; Vogel et al. 2006). If performance generally increases with additional encoding time for all stimuli, this would cast doubt on almost all research claiming to measure limits in working memory - instead suggesting that so-called capacity limits of working memory may actually arise primarily from capacity limits at encoding or during perception (e.g., Stojanoski et al. 2019). Thus, whether and how encoding time influences working memory performance for different kinds of stimuli is central not just to understanding how working memory capacity is affected by stimulus type, but the nature of working memory capacity limits more broadly.

In sum, there are several discrepant results on how encoding time and meaning affect working memory performance, with the majority of studies using meaningless and often simple stimuli claiming that encoding time past a few hundred milliseconds does not affect visual working memory performance (e.g., Luck \& Vogel, 1997; Alvarez \& Cavanagh, 2004; Bays et al. 2011; Tsubomi, Fukuda, Watanabe, \& Vogel, 2013); some suggesting that longer encoding times increase working memory performance for both simple stimuli and real-world objects (Quirk et al 2020; Li et al. 2020), and others reporting selective benefits of long encoding times for realworld objects only (Brady et al., 2016). These mixed results depict the lack of clarity on a seemingly basic issue in the working memory literature. They raise the question of why results differ so greatly among studies, and demand a deeper understanding of the processes involved during working memory encoding, as a function not just of encoding time but of how people process the stimuli.

It is unlikely there is only one reason for the discrepant results with respect to real-objects in particular. For example, in other recent work, we have shown that a major reason not all studies have found object benefits at long encoding times (e.g., Quirk et al. 2020; Li et al. 2020) is that they chose foils unfairly, in a way that disadvantages objects relative to colors (Brady \& Störmer, 2020). Once this is accounted for, significant benefits for memory performance with real-world objects emerge reliably compared to both colors (Brady \& Störmer, 2020) and perceptually-matched meaningless stimuli (e.g., Brady \& Störmer, 2020; Stojanoski et al. 2019; Veldsman et al. 2017; Sahar et al. 2020). In addition, verbal re-encoding is always a potential 
concern with long encoding times, and studies have differed in how they have prevented this (e.g., Quirk et al. 2020, Li et al. 2020; Brady et al. 2016).

In the current work, we investigate additional reasons why object benefits may appear somewhat heterogeneous: we show that longer encoding time is simply one possible way to allow for deeper processing of each of the to-be-remembered stimuli, and argue that any form of deeper processing is particularly beneficial for more meaningful stimuli. We propose that in some situations (or in some instruction conditions), participants may be prone to try to encode the entire display holistically (i.e., in a quick 'snapshot') rather than process the items individually. If this encourages participants to treat objects simply as meaningless colored 'blobs', rather than process them at a high-level, connecting them to prior knowledge, this would clearly reduce the ability to find benefits in memory capacity for meaningful stimuli. Thus, in a series of experiments we directly test whether real-world objects, colors, perceptually-matched less-meaningful objects, and fully scrambled objects benefit from a deeper processing, while manipulating the degree of such deeper processing. To do so, we systematically vary the presentation format of stimuli at encoding to be either simultaneous - encouraging a parallel, 'take-a-quick-snapshot' strategy (similar to how we imagine participants do the task at short simultaneous encoding) - or present the stimuli sequentially, promoting a serial, each-item-atonce strategy.

We find that real-world objects result in higher memory performance than simple stimuli across all experiments (9 total replications of the object benefit in 4 experiments), in support of the account that working memory capacity is higher for meaningful and real-world objects relative to meaningless simple colors and meaningless perceptually-matched stimuli. We also find that real-world objects - and to a lesser degree lightly scrambled versions of the objects - benefit from the sequential encoding and thus deeper, focused-on-individual-items processing, while colors and fully scrambled objects do not. Thus, our results demonstrate that different encoding situations during working memory tasks - previously only indirectly manipulated by changing encoding times - play an important role in constraining working memory capacity, and suggest that different encoding situations can have differential effects for different stimulus sets. Most broadly, our results indicate that single feature objects may be an outlier, not representative of real-world memory situations: due to their unique role in feature-based attention, such stimuli are unlike any realistic stimuli in their affordance of parallel, holistic encoding. Thus, in more realistic situations, memory likely nearly always benefits from in-depth processing of objects 
(e.g., in higher-level visual areas) rather than processing them solely in terms of their low-level features (in a quick 'snapshot').

\section{Experiment 1: How does the memory benefit for objects relative to colors interact with encoding strategy?}

Single colors and other simple features can be processed quickly and in parallel (Treisman \& Gelade, 1980; White et al., 2017), and people tend to make use of ensemble encoding, grouping and other strategies when encoding such simple features into memory (e.g., Brady \& Alvarez, 2015a). By contrast, object recognition is more serial (e.g., Rousselet et al. 2004), or at least severely bottlenecked, with objects benefiting from being individually selected to deeply process them and subject to severe limits from visual crowding (Whitney \& Levi, 2011). Thus, we hypothesized that typical visual working memory studies may be the least advantageous situation for eliciting benefits for encoding meaningful objects: such studies use simultaneous presentations of many stimuli at once, with all being equally task relevant. In the extreme, if people attempt to encode the entire display at once, effectively treating the stimuli as 'colored, textured blobs' without processing their meaning at all, it could even be possible to eliminate object benefits. In contrast, presenting a set of colored circles all at once, which would encourage participants to 'zoom out' and take a 'snapshot' of the entire scene, might encourage the use of global feature-based attention (e.g., Treisman \& Gelade, 1980; White et al., 2017), leading to ensemble processing and chunking of the features (e.g., Brady \& Alvarez, 2015a; Nassar, Helmers, \& Frank, 2018) that would be particularly beneficial for color memory and other low-level features that can easily be processed in parallel.

Thus, we hypothesized that although there are object benefits even in studies with simultaneous presentations of a large number of items (i.e., Brady \& Störmer, 2020), such conditions may nevertheless be among the most favorable conditions for simple stimuli and least favorable for realistic meaningful stimuli. In real-world scenarios where participants use visual working memory to perform tasks (e.g., holding in mind the target of an eye movement: Hollingworth et al. 2008; or the target of an action: Ballard et al. 1995; Hayhoe et al. 2003), they are relatively unlikely to try to equally encode many stimuli at once, and instead to focus additional resources on more task-relevant stimuli (e.g., Salahub et al. 2019) and encode stimuli sequentially (e.g., Ballard et al. 1995). 
In the current experiment we sought to directly test the hypothesis that real-world objects, but not colored circles, benefit from a serial, item-based encoding. To do so, we compared memory performance across two encoding scenarios for objects and colors: We either presented items simultaneously, encouraging a parallel processing strategy, or sequentially, encouraging a serial processing strategy, while keeping the amount of time each item could be processed constant. We reasoned that this was a direct manipulation of different encoding strategies, but was likely similar to the difference that is tapped indirectly when encoding times are manipulated: with longer encoding times generally facilitating serial, item-based processing, and short encoding times - or even the possibility of short encoding times - generally encouraging simultaneous, parallel processing of the items.

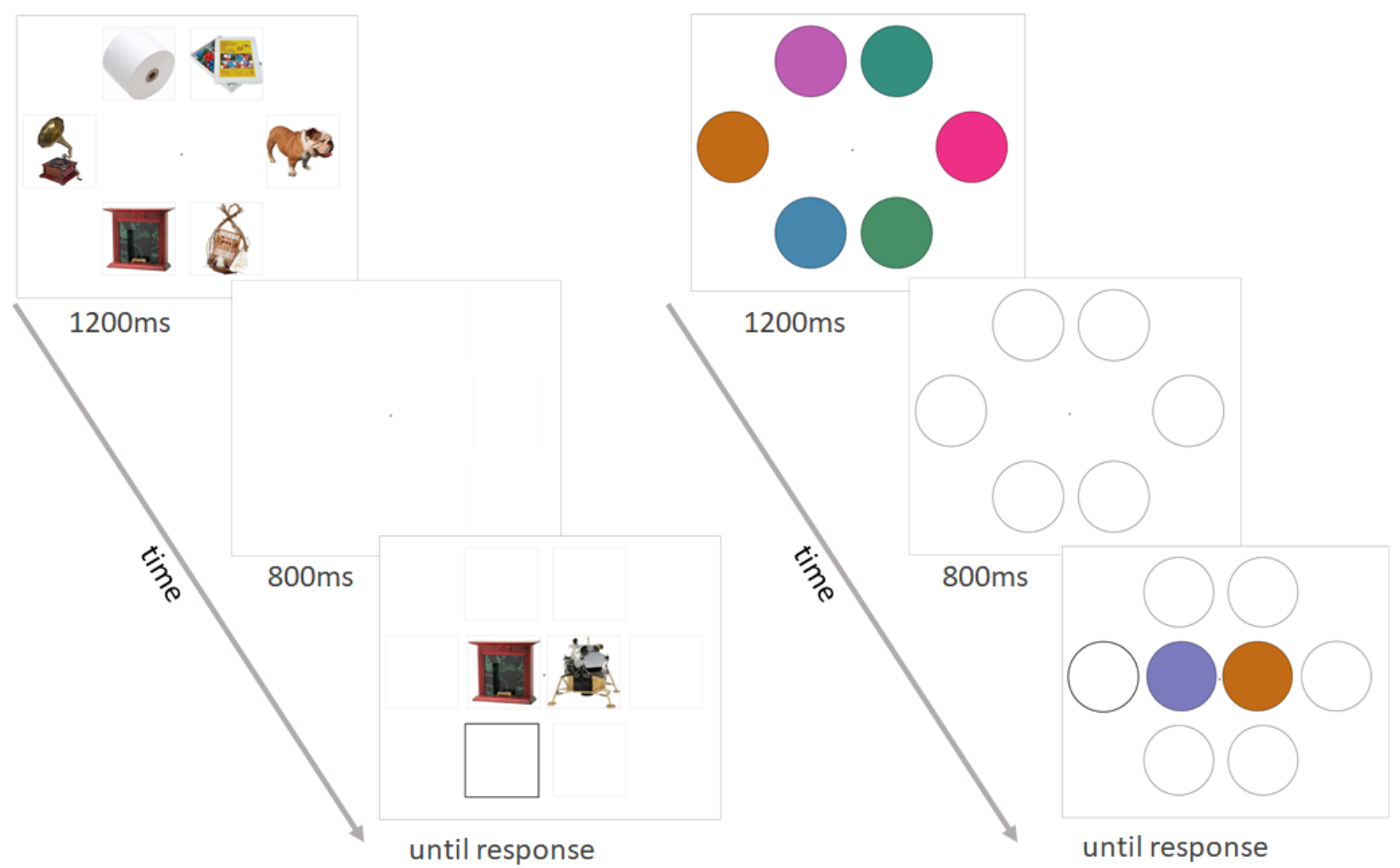

Figure 1. In all experiments, participants saw either 6 real-world objects or 6 colors and had to remember them over a brief delay, followed by a 2-AFC memory test. In simultaneous encoding conditions (shown), they saw all the objects at once. In sequential encoding conditions, objects appeared at the same spatial locations and in a spatially and temporally predictable sequence, but for 200ms each, and they were probed in the same way.

\section{Method}

The study design, hypothesis, analysis plan and exclusion criteria were preregistered: 
https://aspredicted.org/blind.php?x=gh9md2. Materials and data are available at: https://osf.io/va2te/

Participants: Fifty participants were used in the final data analysis. Data from 4 participants were excluded and replaced per pre-registered exclusion criteria. All participants were run in the laboratory and gave written informed consent prior to starting the experiment as approved by the Institutional Review Board at UC San Diego.

Power. At long encoding times as in the current study, previous work (Brady et al. 2016) found the difference between object performance and color performance (in terms of d') had an effect size of $d_{z}=0.74$. We had twice as many trials per condition, which would be expected to increase this effect size significantly, but at the same time, effects tend to be smaller in replications, and other work has disputed this effect (e.g., Quirk et al. 2020). Thus, we used this effect size as-is to calculate our power. By this calculation, the current study had $99.9 \%$ power to find this effect. We were also interested in the potential interaction, which would have twice as much variability in its estimate, halving the effect size. We thus had approximately $73 \%$ power to detect such an interaction.

Stimuli and Procedure: We contrasted memory for objects and colors in a working memory task modeled after Brady et al. (2016). The memory task used a 2-AFC memory probe (which of these two items did you see?) to allow us to control foil similarity and avoid the need to model response criterion differences.

For colors, we used a standard color circle (Suchow et al. 2013; Schurgin et al. 2018) of radius 49 in the CIE $L^{*} a^{*} b$ space (centered at $L=54, a=21.5, b=11.5$ ). Both shown items and test foils were required to be a minimum of $15^{\circ}$ apart on the color wheel to reduce chunking and grouping across items. Target colors and test foils were chosen to be maximally dissimilar $\left(180^{\circ}\right.$ apart on the color wheel).

For object stimuli, we used the Brady et al. (2008) object image database, as in Brady et al. (2016). We aimed to exactly replicate Brady et al. (2016), by using their particular set of objects and foils (categorically distinct and hand-pruned to be visually distinct; see publicly available materials). 
Participants remembered 6 colors or 6 real-world objects on each trial. Stimuli were either presented simultaneously for $1200 \mathrm{~ms}$, or one-at-a-time for $200 \mathrm{~ms}$ each (followed by a $200 \mathrm{~ms}$ inter-stimulus interval (ISI)). In sequential conditions, the items always appeared in the same order (clockwise starting at $9 \mathrm{pm}$ ) making them strongly temporally and spatially predictable. The long encoding time, combined with fixed spatial positions with placeholders present during the delay helped ensure there was little to no location noise that can cause misbinding.

After a delay $(800 \mathrm{~ms})$, a location probe was shown to indicate which location was being probed, and two test stimuli appeared in the center of the screen. Participants performed a 2-AFC, indicating which of the two stimuli appeared at the probed location during encoding (Figure 1). This required information about exactly which object was at a particular location on this particular trial.

We used a within-subject design such that each participant encoded colors simultaneously, colors sequentially, objects simultaneously, and objects sequentially. Conditions were blocked (4 blocks overall) and their order counterbalanced across participants subject to the constraint that participants either did both simultaneous conditions first (order counterbalanced) or both sequential conditions first. We counterbalanced in this way as we hoped to encourage participants to apply the same encoding strategy for both stimuli sets in each of the sequential and simultaneous conditions. Participants completed 60 trials of each condition.

To further ensure that any object benefit was not caused by verbal encoding, during the entire experiment, participants performed a concurrent articulatory suppression task designed to prevent them from verbally encoding any of the items. In particular, they were required to say "the" out loud continuously for the entire duration of the experiment, and this was monitored by an experimenter throughout the experiment.

Analysis. Working memory performance was quantified using d' for a 2-AFC task, $[z H-z F A] / \sqrt{2}$ where $P$ is percent correct and $\Phi$ is the Gaussian cumulative distribution, $z H=\Phi(P)$ and $z F A=$ $\Phi(1-P)$. Per the preregistration, data were excluded if the d' averaged across all conditions was below 0.5 , or if greater than $10 \%$ of individual trials were excluded. Individual trials were excluded if: 1) A response occurred less than $150 \mathrm{~ms}$ after the response screen appeared. 2) The response occurred more than 5 s after the response screen appeared. 


\section{Results}

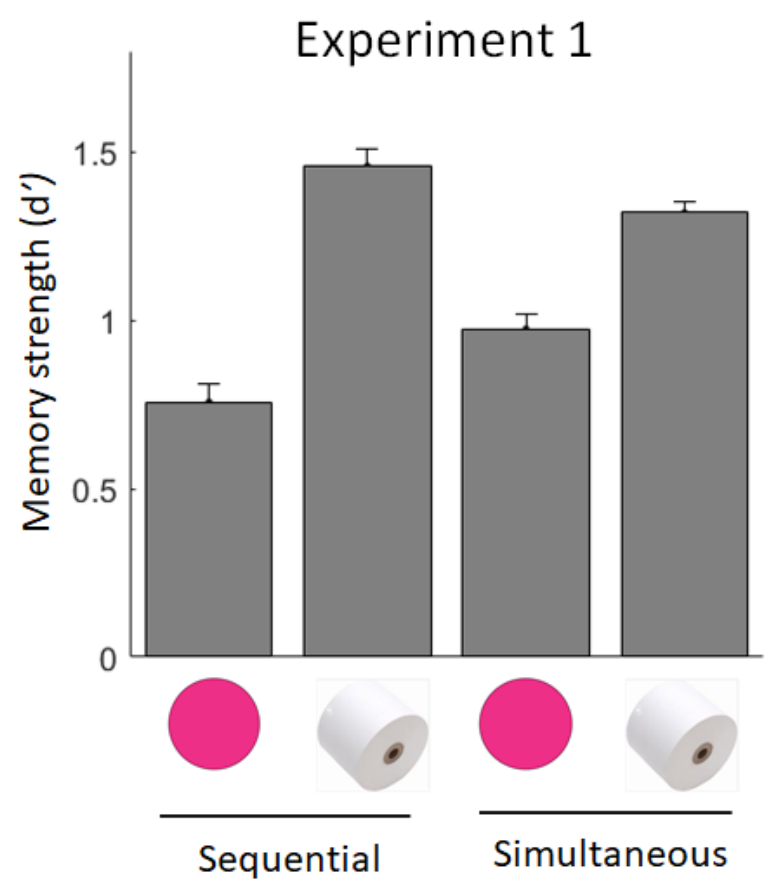

Figure 2. Results of Experiment 1. Memory performance for objects was overall higher than for colors, but there was a qualitative difference between the two stimuli in terms of encoding strategy: objects show a larger benefit from being encoded sequentially, while colors show a benefit in being encoded simultaneously.

We found a main effect of stimulus type, indicating that objects were remembered better than colors overall $\left(F(1,49)=88.275, p<0.0001, \eta_{p}^{2}=0.64\right)$. There was no main effect of encoding type (simultaneous vs. sequential), $F(1,49)=0.572, p=0.45, \eta_{p}^{2}=0.015$, but there was an interaction between encoding type and stimulus type $\left(F(1,49)=12.114, p=0.001, \eta_{p}{ }^{2}=0.20\right)$, such that objects were remembered better during sequential encoding and colors were better remembered when encoded simultaneously (Figure 2). Follow-up pairwise comparisons confirmed this: When objects were encoded sequentially, memory performance was higher than when encoded simultaneously $t(49)=2.19, p=0.033, d_{z}=0.31$; but when colors were encoded sequentially, memory performance was lower than when colors were encoded simultaneously $\left(t(49)=-2.65, p=0.011, d_{z}=0.37\right)$. 
This pattern of results is consistent with our hypothesis that simple features - such as colors can be processed efficiently in parallel, likely benefitting from ensemble and chunking processes when shown at the same time; while real-world objects benefit from a deeper item-based processing that is facilitated by sequential encoding, where each item can be focused on one at a time. Notably, the crossover interaction we found suggests a qualitative difference in the best way for people to encode meaningful stimuli vs. simple features. Given existing evidence suggestive of different mechanisms available only for simple features (e.g., feature-based attention), this raises the strong possibility that the models researchers have developed to explain memory capacity in simple features (particularly at high set sizes, where parallel encoding is necessary) may not apply at all to more meaningful objects or more realistic situations where visual working memory is used.

\section{Experiment 2: The role of spatial location: Do objects in particular benefit from deeper processing afforded by sequential presentations, and is this impacted by spatial locations being present?}

To replicate our results from Experiment 1 of sequential encoding boosting memory performance for real-world objects, and to eliminate the possibility of any practice or strategy effects that could arise in a within-subject design, Experiment 2 was a between-subject version of that experiment. This helps eliminate the concern that participants may have adapted to one encoding strategy based on what they were exposed to first and continued to use it even when the presentation format changed. For example, if a participant began with the sequential presentation that encourages encoding each item separately, then they may continue focally attending to each item during simultaneous presentation trials (or vice versa). In this experiment we also increase the encoding time in the simultaneous condition to be greater than in the sequential conditions (1200 vs. $2000 \mathrm{~ms}$ ), to reduce the possibility that items are encoded somewhat longer during the sequential condition because participants may continue to process them during the time between objects (200ms ISI) - which could, at least in theory, differentially impact objects vs. colors. Finally, Experiment 2 also added a new condition in which we presented all items sequentially at the center of the display, instead of presenting them at six different locations. This allowed us to test the role of spatial information during encoding and provided another test for the robustness of the object benefit. 


\section{Method}

Materials and data are available at: https://osf.io/va2te/.

Participants: Fifty unique US-based participants from Amazon's Mechanical Turk were in the final data set of each of the three across-participant experimental groups (total 150 participants; all with $>=95 \%$ previously accepted HITs). An additional 10 participants were excluded in the non-spatial sequential condition; 15 excluded in the spatial-sequential condition; and 13 excluded in the simultaneous condition. Exclusion criteria at the subject level were performance below chance or $10 \%$ of trials excluded; and trials were excluded based on the same rules as previously (reaction times $<150 \mathrm{~ms} ;>5,000 \mathrm{~ms}$ )

Stimuli and procedure: In all conditions, participants performed 120 trials, 60 with color and 60 with real-world objects. Stimuli and procedure for each trial were identical to Experiment 1 with the following exceptions: Encoding type was varied across participants, such that group 1 only performed trials in which items were presented sequentially (200ms each with a $200 \mathrm{~ms}$ ISI) at the center of the screen; group 2 only performed trials in which the items were presented sequentially at distinct spatial locations (200ms each with a $200 \mathrm{~ms}$ ISI); and group 3 performed trials in which the items were presented simultaneously. The simultaneous presentation used a longer encoding time than in Experiment 1 (2000ms), since we reasoned it is possible participants continue to process the stimulus during the ISI period in the sequential condition, an advantage they would not have with $1200 \mathrm{~ms}$ encoding in the simultaneous condition. The first condition thus removed spatial cues from encoding, a new condition in this experiment, and the third condition examined whether objects continue to benefit from sequential encoding even with a longer encoding time in the simultaneous condition. Participants performed the same verbal suppression task as in Experiment 1, but in this study, unlike Exp. 1, compliance was not monitored continuously by the experimenter.

\section{Results}

Our results in the sequential (spatial) and simultaneous conditions replicate the results of Experiment 1 (Figure 3). We also find that the sequential non-spatial condition, where items were all presented at the center of the screen, was similar to the results from the sequential (spatial) condition. 
In particular, in all 3 conditions taken individually, there was a reliable and large object benefit: $t(49)=12.17, p<0.001, d_{z}=1.72$ (sequential, center); $t(49)=11.43, p<0.001, d_{z}=1.62$ (sequential, spatial); $\mathrm{t}(49)=5.36, \mathrm{p}<0.001, \mathrm{~d}_{z}=0.76$ (simultaneous).

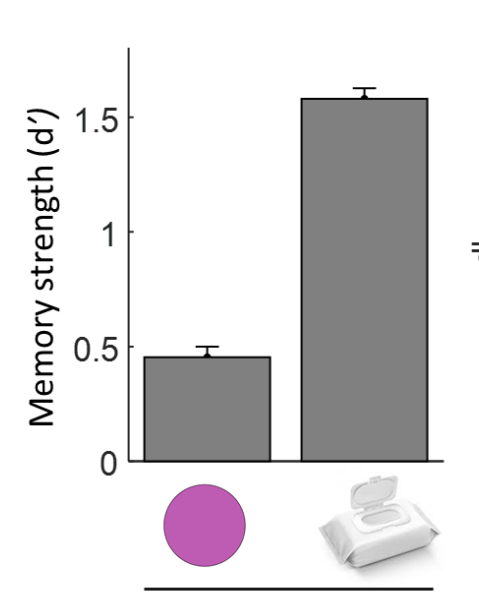

Sequential (center)

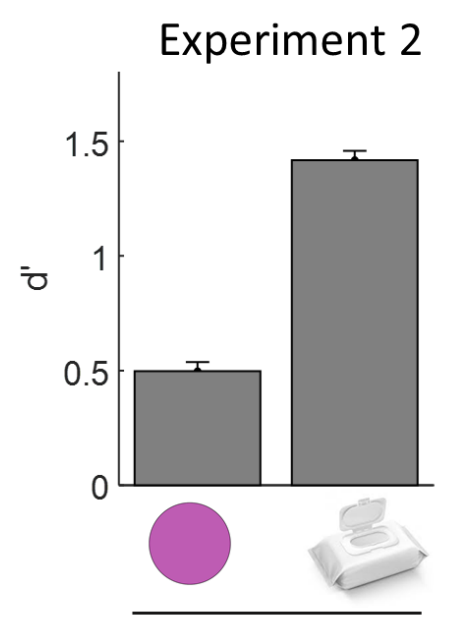

Sequential (spatial)

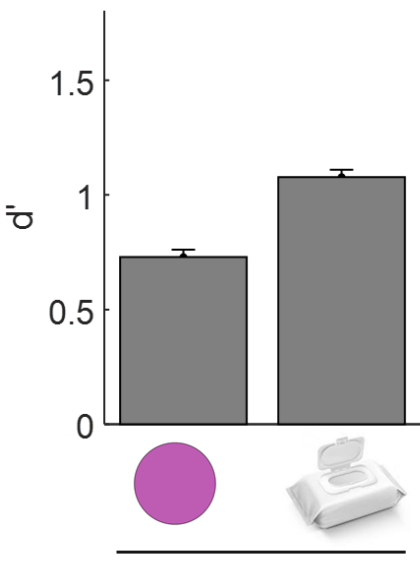

Simultaneous (2 sec)

Figure 3. Results of Experiment 2. We replicate reliable object benefits in all conditions. We also replicate the cross-over effect found in Experiment 1 where objects are better remembered in sequential encoding conditions whereas colors are best remembered when presented simultaneously.

We also replicated the dissociable effects of sequential and simultaneous encoding for objects vs. colors observed in Experiment 1, even with the longer encoding time in the simultaneous condition. In particular, colors were better remembered when presented simultaneously than sequentially (spatially), $t(98)=2.89, p=0.005$, Cohen's $d=0.58$, and better remembered when presented simultaneously than sequentially (at the center), $t(98)=3.36, p=0.001, d=0.67$. By contrast, objects were remembered better when presented sequentially (spatially) than simultaneously, $t(98)=3.02, p=0.003, d=0.60$, and also better sequentially (central) than simultaneously, $t(98)=4.43, p<0.001, d=0.89$. The difference in performance between objects in the two sequential conditions was not statistically significant $(t(98)=1.30, p=0.198, d=0.26)$.

Thus, taken together, the data from Experiment 2 suggest that regardless of spatial location availability, objects show an extremely large benefit over colors when items are encoded sequentially, and a smaller (though still large) benefit when items are encoded simultaneously. They also replicate the qualitative difference in encoding between the two stimuli types: even with longer encoding time in the simultaneous condition than sequential conditions, objects benefit more from sequential whereas colors benefit more from simultaneous encoding. 


\section{Experiment 3: Is the overall object benefit purely from stimulus complexity, or a result of knowledge/familiarity? Lightly scrambled objects.}

Experiments 3 and 4 address two questions. First, they examine the role of semantics in the object benefit from Experiments 1 and 2 and Brady et al. (2016) and Brady and Stoermer (2020). One possibility is that the object benefit is simply a 'complex' stimuli benefit - that is, stimuli that are more complex and have 'more' features are better remembered regardless of the meaningfulness of those features. This could arise if there are fixed pools of resources for each basic feature (e.g., Bays et al. 2009), and real objects can benefit from recruiting multiple such pools of resources whereas colors cannot. Such a hypothesis is superficially at odds with work showing worse performance for complex but meaningless stimuli than simple stimuli (e.g., Alvarez \& Cavanagh, 2004; Brady \& Alvarez, 2015b), and work using perceptually-matched but non-meaningful stimuli (e.g., Asp et al. 2019; Stojanoski et al. 2019; Sahar et al. 2020), but is important to address directly.

In addition, these experiments also address the question of sequential vs. simultaneous encoding in complex, meaningless stimuli. In particular, Experiments 3 and 4 ask whether the sequential processing benefit found in Exp. 1 is unique to objects, or dependent on how much meaningful information can be extracted from the stimuli. We hypothesized that stimuli that provide significant semantic information when processed more deeply may benefit from deeper processing - since additional informative item-specific information can be extracted - and that stimuli that are semantically meaningless (just 'colored blobs') would not benefit from such processing. This would be consistent with our hypothesis that stimuli made up solely of meaningless bundles of color and orientation may be relatively unique in their affordance of parallel processing/ensembles/grouping - continuing the theme from Experiments 1 and 2 that such stimuli are perhaps not a good case study of memory as they are (uniquely) supported by feature-based attention (Treisman \& Gelade, 1980).

To test this, in Experiment 3, we compared memory for real-world objects relative to lightly scrambled versions of these objects - images of these objects where one side was vertically flipped, preserving some meaning and recognition ability but reducing meaningfulness compared to real objects (see Appendix). In Experiment 4, we compared real-world objects to 
fully scrambled versions of these objects, which were effectively just colored blobs without meaning - but contained just as many and complex visual features as the real objects. We validated the effects of these manipulations on meaningfulness in a separate experiment (see Appendix). The light scrambling we use in Experiment 3 is purposefully an extremely subtle manipulation of the objects. It has been shown to distort the meaningfulness and familiarity of objects as well as affect memory performance (Shoval \& Makovski, 2019), but preserves a fair amount of the meaning of the objects (Appendix). By contrast, our fully scrambled objects in Experiment 4 used diffeomorphic scrambling to remove effectively all ability to recognize the objects (Stojanoski \& Cusack, 2014), massively reducing their meaningfulness, while still maintaining visual complexity (Appendix).

Note that the light scrambling condition, on its own, is therefore a strong test of the role of knowledge and familiarity in working memory, as though less meaningful, these stimuli are visually extremely similar to the meaningful real objects (see Fig. 4) and still preserve significant semantic recognition (Appendix). Thus, any benefit for real objects over these in memory suggests a major role for meaningful processing, given they are nearly identical in visual features to the original objects.

Overall, in Experiments 3 and 4, we predicted that like real objects lightly scrambled but still meaningful objects would benefit from deeper processing; but that fully scrambled objects, that are effectively just colored blobs with no deeper processing possible, would benefit from parallel processing like simple features. We also expected a general memory benefit for real-world objects relative to both the lightly and fully scrambled versions of these objects.

\section{Method}

Materials and data are available at: https://osf.io/va2te/

Participants: The final data set consists of 30 participants tested in person at UC San Diego. Data from three additional participants were excluded per the same rules as the previous experiment. We had planned 50 participants to match Exp. 1, but data collection was interrupted by COVID-19.

Power. We hoped to power the current study to detect a main effect in performance between meaningful and scrambled objects, as well as an interaction in encoding benefits for sequential 
vs. simultaneous if present. Using the results from Exp. 1 revealed that even with only the dataset of 30 participants, if the difference between meaningful and non-meaningful objects was half as large as that between meaningful objects and colors, we had $>99 \%$ power to detect such an effect with this sample, as well as $77 \%$ power to detect an interaction of the size observed in Experiment 1. Thus, we analyzed the data with this sample.

Stimuli and Procedure: The experimental set-up was the same as Experiment 1 with the only exception that we used lightly scrambled-objects instead of colors. Thus, the experiment consisted of four conditions: real-world objects simultaneous; lightly-scrambled-objects simultaneous; real-world objects sequential; lightly-scrambled-objects sequential. For the lightlyscrambled-object conditions, the same object database was used as for the intact objects, but either the left or right half of the object were flipped vertically, making it more difficult to recognize the object (Shoval \& Makovski, 2019), while simultaneously keeping the objects nearly identical in their visual complexity and visual features.

For each participant we randomly assigned which objects would be seen as lightly scrambled and which objects would be seen as intact, such that no object was used across both conditions for an individual subject. The test foils were chosen to be categorically and visually dissimilar, just as in Experiment 1; i.e., we used the same object pairs as Brady et al. (2016), with or without scrambling both items. Furthermore, participants again concurrently performed a verbal interference task throughout the experiment - articulatory suppression by saying 'the' out loud for the entire duration of the study that was continuously monitored by an experimenter. 


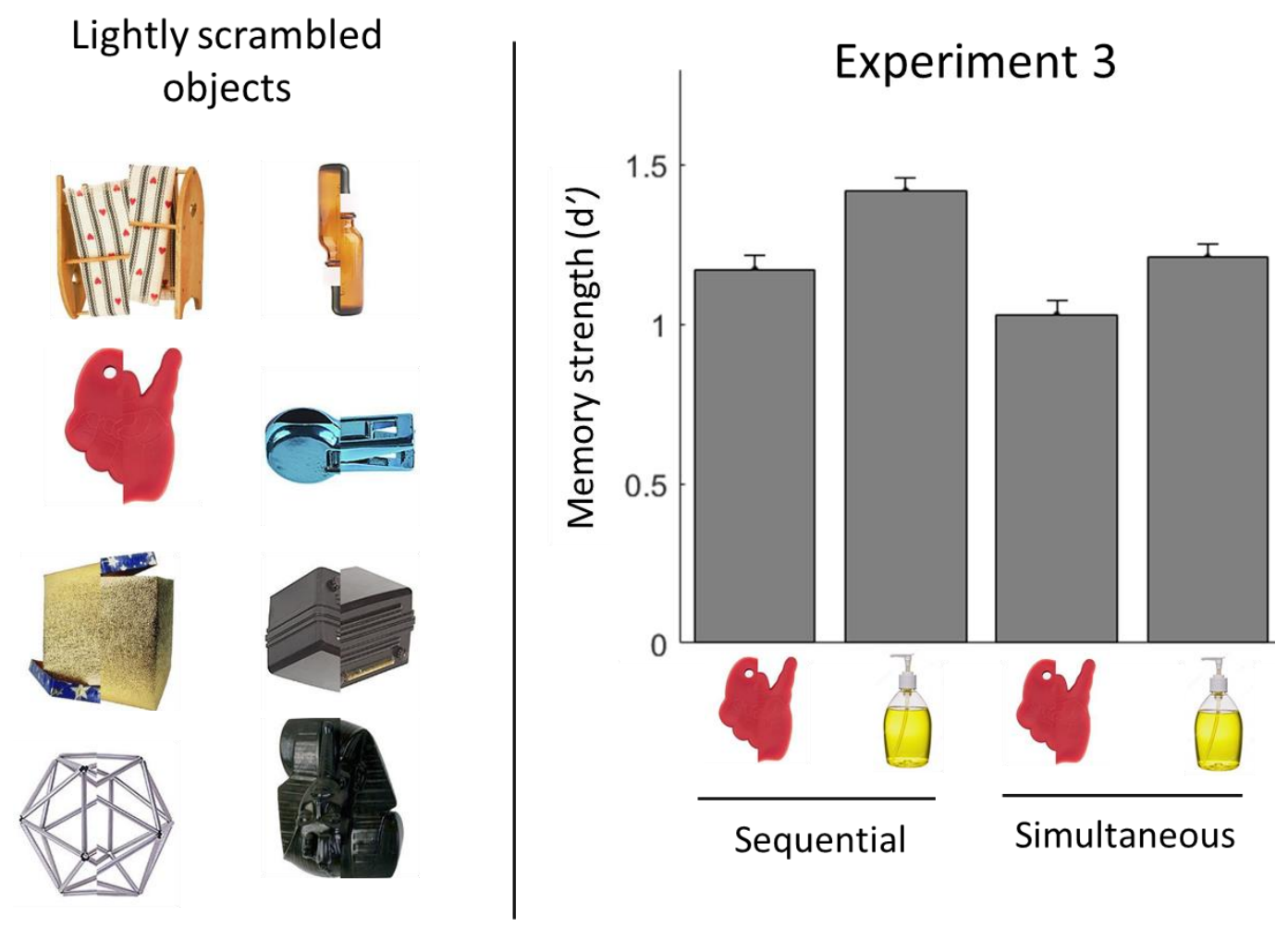

Figure 4. Stimuli and results of Experiment 3. Objects were lightly scrambled by flipping vertically one half of the object. Real-world objects resulted in overall higher memory performance relative to lightly-scrambled objects, and both lightly-scrambled objects and intact objects showed higher memory performance when presented sequentially relative to simultaneously.

\section{Results}

We observed a main effect of stimulus type, such that intact objects showed higher memory performance than scrambled versions of these objects $F(1,29)=15.722, p=0.0004, \eta_{p}{ }^{2}=0.35$, replicating a meaningful object benefit in working memory (Figure 4). Furthermore, we found a benefit of a sequential encoding for both, $F(1,29)=12.755, p=0.0013, \eta_{p}{ }^{2}=0.31$, and no interaction $\left(F(1,29)=0.595, p=0.45, \eta_{p}{ }^{2}=0.02\right)$. Follow-up pairwise comparisons confirmed that the sequential encoding benefit was reliable for each stimulus type: For intact objects, sequential encoding resulted in higher memory performance relative to simultaneous encoding $\left(t(29)=3.43, p=0.002, d_{z}=0.63\right)$; and for scrambled objects, memory performance was also higher for sequential relative to simultaneous encoding $\left(t(29)=2.07, p=0.048, d_{z}=0.38\right)$.

These data are consistent with our predictions that to some extent any meaningful stimuli - i.e., real-world objects and also their lightly-scrambled counterparts - benefit from sequential encoding and thus from deeper and more serial processing. Furthermore, these results replicate 
the general advantage for real-world objects (Brady et al., 2016), even compared to extremely visually similar stimuli. Thus, they also provide a conceptual replication of the results from Asp et al. (2019) which show enhanced memory performance and enhanced active neural storage (via the $\mathrm{CDA}$ ) for meaningful stimuli compared to perceptually-matched stimuli that are not subjectively seen as meaningful (Mooney faces), as well as other similar work (e.g., Stojanoski et al. 2019).

\section{Experiment 4: Is the overall object benefit purely from stimulus complexity, or a result of knowledge/familiarity? Fully scrambled objects.}

In Experiment 4, we compared real-world objects to fully scrambled objects, which were effectively just colored blobs without meaning (see Appendix for experimental validation of their lack of meaningfulness). In contrast to Experiment 3, we predicted that fully scrambled objects, that are effectively just colored blobs with no deeper processing possible, would benefit from parallel processing like simple features - rather than from deeper processing like the lightly scrambled objects in Experiment 3. We thus predicted they would be better remembered in simultaneous than sequential conditions.

Because these objects are similarly complex and contain many features, like real objects, this experiment thus provides a useful test of whether the meaningfulness of the stimuli is the critical aspect that leads to enhanced object memory with sequential presentations. If instead some aspect of visual complexity itself is responsible for the sequential benefit - i.e., perhaps eliminating visual crowding (Whitney \& Levi, 2011) is more important for real objects than simple stimuli - than there should be a sequential benefit even for these almost totally meaningless objects because of their visual complexity.

\section{Method}

Materials and data are available at: https://osf.io/va2te/

Participants: The final data set consists of 50 participants. This experiment was run on UCSD undergraduates, but the data was collected online due to COVID-19. 6 additional participants were excluded according to the same rules as the previous experiments. 
Stimuli and Procedure: The experimental set-up was the same as Experiment 3 with the only exception that we used fully scrambled-objects instead of lightly-scrambled objects. Thus, the experiment consisted of four conditions: real-world objects simultaneous; fully-scrambledobjects simultaneous; real-world objects sequential; fully-scrambled-objects sequential. For the fully-scrambled-object conditions, the same object database was used as for the intact objects, but used diffeomorphic scrambling to remove effectively all ability to recognize the objects (Stojanoski \& Cusack, 2014; see Appendix for recognition data). Diffeomorphic scrambling is done by repeatedly applying a 2D- flow field to the images, with random phase and amplitude, effectively distorting the image without changing any major features of the overall distribution of pixels. It is designed to match the visual system response between the original and scrambled images in early stages of visual processing, while removing the ability for participants to recognize the images. It has no duplication of removal of parts, meaning it, for example preserves the topography of the image, such that if there are a certain number of islands of pixels surrounded by white in the original images, the same is true on the transformed images (Stojanoski \& Cusack, 2014).

As in Experiment 3, for each participant we randomly assigned which objects would be seen as scrambled and which objects would be seen as intact, such that no object was used across both conditions for an individual subject. The test foils were chosen to be categorically and visually dissimilar, just as in Experiment 1. Furthermore, participants again concurrently performed a verbal interference task throughout the experiment - articulatory suppression by saying 'the' out loud for the entire duration of the study. 


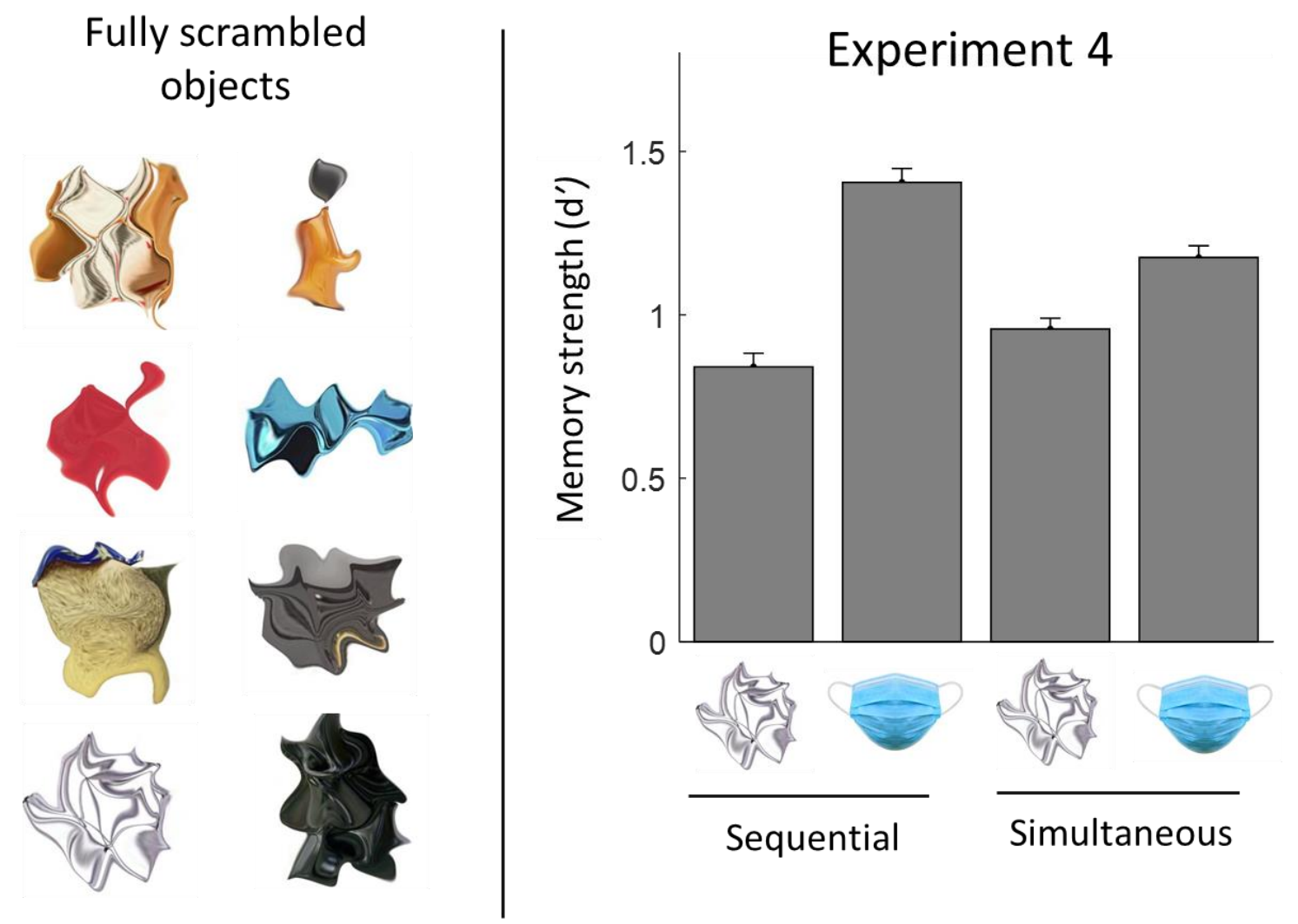

Figure 5. Stimuli and results of Experiment 4. Objects were fully scrambled to remove the ability to recognize them while preserving relevant low and mid-level image statistics. Real-world objects resulted in overall higher memory performance relative to fully-scrambled objects. Like colors, fully scrambled objects were better remembered when presented simultaneously; whereas intact objects showed higher memory performance when presented sequentially relative to simultaneously.

\section{Results}

We observed a main effect of stimulus type, such that intact objects showed higher memory performance than fully scrambled versions of these objects $F(1,49)=57.85, p<0.0001$, $\eta_{\mathrm{p}}{ }^{2}=0.54$, replicating a meaningful object benefit in working memory. There was no main effect of sequential encoding $\left(F(1,49)=2.196, p=0.14, \eta_{p}^{2}=0.04\right)$, but there was a crossover interaction $\left(F(1,49)=16.94, p=0.0001, \eta_{p}^{2}=0.26\right)$, such that real-objects were better remembered with sequential encoding and very scrambled objects were better remembered with simultaneous encoding. Follow-up pairwise comparisons confirmed that the differences in encoding were reliable for each stimulus type: For intact objects, sequential encoding resulted in higher memory performance relative to simultaneous encoding $\left(\mathrm{t}(49)=3.86, \mathrm{p}<0.001, \mathrm{~d}_{\mathrm{z}}=0.55\right)$; and for fully scrambled objects, memory performance was higher for simultaneous relative to sequential encoding $\left(t(49)=2.13, p=0.039, d_{z}=0.30\right)$. 
Taken together, Experiments 3 and 4 provide strong evidence that the potential for extracting meaning from a stimulus determines whether it benefits from sequential processing. On the one extreme, features like single colors have an incredible affordance of parallel processing; at the opposite extreme, real objects have significant additional semantic information that can be extracted when they are processed as individuals, improving memory in sequential conditions. Experiments 3 and 4 show there are also gradations: both kinds of reasonably meaningful stimuli - i.e., real-world objects and also their lightly-scrambled counterparts - benefit from sequential encoding and thus from deeper and more serial processing; whereas fully scrambled, unrecognizable stimuli and simple features like color benefit most from simultaneous processing. Experiment 4 shows that the sequential benefit is not solely due to visual complexity but is related to the meaningfulness of the stimuli.

Overall, these experiments suggest that results from simple features at high set sizes should not be generalized to real-world objects, or even to more complex but still meaningful stimuli, like lightly scrambled versions of objects. Using simple, unidimensional stimuli like colored circles or oriented bars may not just chronically underestimate working memory performance but also provide qualitatively incorrect conclusions about how memories are best formed and stored in more realistic situations. $z$

\section{General Discussion}

Across a series of experiments, we found higher working memory performance for real-world objects relative to simple colors and scrambled objects. This was true even in situations that are suboptimal for this object benefit to emerge, like the simultaneous presentation of all stimuli. Such conditions resulted in only moderate, but nonetheless robust, benefits for meaningful objects in comparison to simple colors and scrambled stimuli. Conditions that encouraged 'deeper' item-specific processing - i.e. the sequential presentation of stimuli — resulted in reliably larger benefits for meaningful stimuli, whereas such conditions uniquely disadvantaged simple colors and meaningless stimuli compared to conditions that facilitated parallel encoding (i.e., simultaneous conditions). Objects were better remembered when people were forced to focus on them one at a time, even though in the simultaneous condition we used relatively long encoding times ( $>1 \mathrm{~s}$, and up to $2 \mathrm{~s}$ ), to match how much time each item could be processed across both conditions, thus allowing the possibility of participants' serially moving attention 
during the encoding period. With extremely short encoding times, which even more strongly encourage a single 'snapshot' of the display rather than individualized encoding, this difference would be expected to be even further exacerbated.

Altogether, these data support a model of working memory where capacity depends on the type of information that is being remembered and how it is encoded. They suggest that using simple stimuli may be causing us to chronically underestimate working memory capacity, as well as promote the study of mechanisms of working memory storage that are unique to simple stimuli and not generalizable to complex, meaningful stimuli that we actually encounter in the world. In particular, stimuli consisting solely of meaningless visual features like color may encourage global, feature-based attention encoding strategies that are not nearly as useful as in-depth processing for realistic objects.

\section{Comparing working memory across stimulus sets on common ground}

Why have some other studies not found a working memory benefit for objects relative to colors? We believe there are a number of sources that likely underlie the discrepancies across studies. First, in another recent manuscript we demonstrate the importance of comparing memory performance across stimulus sets on common ground by using comparable target/foil similarity at test (Brady \& Störmer, 2020). Earlier work has shown that the similarity between target and foil critically determines performance (e.g., Awh et al. 2007, although see Brady \& Alvarez, 2015b), and this issue has most recently been quantified in the Target Confusability Competition (TCC) model of working memory (Schurgin, Wixted, Brady, 2020), which shows that even seemingly large differences between items and foils in a given stimulus space may not be sufficient to avoid underestimating working memory capacity, as confusability between items is not at floor even for large target/foil changes. Thus, it is of tremendous importance to maximize the dissimilarity of the memory foils for both stimulus sets when comparing working memory capacity.

By contrast, some previous studies that compared working memory capacity for colors and realworld objects used maximally dissimilar colors (i.e., $180^{\circ}$ on the color wheel), but then chose objects randomly from a database of many objects, with varying levels of visual and semantic similarity (Li et al. 2020; Quirk et al. 2020). However, this does not in any way maximize dissimilarity for objects, effectively disadvantaging them relative to colors, and - at least in large part - explaining why there were no object advantages over colors at long encoding 
times in those studies (Brady \& Störmer, 2020). In the current work, where we chose object foils to be extremely dissimilar from targets, just like colors, we repeatedly replicated Brady et al. (2016)'s finding that objects are better remembered than colors in long simultaneous encoding conditions, strongly contrasting with Li et al. (2020) and Quirk et al. (2020). Thus, one major reason why some labs have not found object benefits is that they did not make target/foil similarity comparable across their stimulus sets.

\section{Different encoding strategies benefit objects and meaningless stimuli differently} A second source of heterogeneity in object benefits - and the main focus of the current study - is how these stimuli are processed at encoding. Specifically, we demonstrate that serial and focused item-based encoding enhances working memory benefits for real-world objects, while distributed parallel encoding facilitates memory for color displays (Exp. 1 \& 2). We also found a serial benefit - albeit smaller — for lightly scrambled objects that still maintained some meaningful semantic information (Exp. 3), but a simultaneous presentation benefit for fully scrambled, meaningless object (Exp. 4). Experiment 4 suggests that the benefit of sequential encoding is not a result of their visual complexity or number of visual features; instead, sequential benefits arise only for stimuli where meaningful information can be extracted when items are individually processed.

Overall, then, the benefit for meaningful stimuli is strongest when participants can encode each item individually, recognize its identity, and connect it to existing knowledge. This is consistent with previous explanations of the object benefit: such benefits to working memory storage do not seem to arise because of more complex visual features, but rather their meaningfulness (Brady et al, 2016; Asp et al., 2019). Thus, effectively visually identical stimuli are better remembered when they can be processed in a meaningful way (e.g., Alvarez \& Cavanagh, 2004; Ngiam et al. 2017; Asp et al. 2019), and semantic knowledge about objects in particular is critical (Starr, Srinivasan, Bunge, 2020).

Under this logic, any conditions that enable deeper processing of items should be particularly beneficial for real-world objects. In other work, we have used long encoding times to facilitate focused item-based encoding, and also found a selective benefit for objects in working memory at long encoding times (Brady et al. 2016, Asp et al., 2019). In the current work, we show this benefit is enhanced by independent item processing rather than simultaneous encoding of many items. 
What then, is the role of encoding vs. maintenance in visual working memory limits? This question is particularly acute given that two recent studies reported benefits of long encoding for both objects and colors (Quirk et al 2020; Li et al. 2020) — seemingly at odds with the selective object benefit we previously reported as well as significantly deviating from important claims over the last 20+ years that have repeatedly argued that working memory for simple stimuli quickly 'fills up' and reaches a fixed capacity limit (e.g., Luck \& Vogel, 1997; Alvarez \& Cavanagh, 2004). We, too, have found that color performance does improve with time (e.g., Schurgin et al. 2020), although as shown in the current work and Brady and Störmer (2020), never reaching the level of object performance. The current work is thus consistent with a growing literature suggesting that encoding differences - either encoding strategy or encoding times - change working memory performance, raising fundamental questions about the purity of the putative fixed capacity "memory" limits claimed in earlier work.

This is particularly important if there is significant variance in encoding strategies. While varying encoding time likely taps into different encoding strategies, such that shorter encoding time on average - encourages parallel processing and longer encoding time encourages serial, item-based encoding, it is not clear that this is always the case, as it is an indirect way to influence how items are being processed. Participants might be able to encode solely the lowlevel visual details of the display, ignoring semantic and higher-level visual information, even when items are presented for a long encoding time. Indeed, individuals are known to vary in their propensity to take on more holistic encoding strategies (Babic et al. 2019; Linke et al, 2011; Cusack et al, 2009), resulting in incorrect estimates of their apparent visual working memory capacity and working memory capacity's relationship to fluid intelligence (Babic et al. 2019; Cusack et al, 2009) based only on encoding strategy differences. Such holistic strategies, as we show in the present study, are advantageous when remembering simple features and it is thus conceivable that such strategies would be used by participants that perform color and object tasks intermixed, or in cases where that parallel 'take-a-snapshot' strategy feels subjectively less effortful. Thus, longer encoding time alone may not always result in a selective object benefit but only when such encoding times are successful in promoting a deeper processing of these items. Importantly, even for simple stimuli, such a strong role for encoding points to the difficulties facing fixed-capacity models of memory performance.

If encoding strategy or encoding times change working memory performance in a relatively smooth way, this raises important questions for all fixed capacity models of visual working 
memory, including those that assume fixed object limits (e.g., Adam et al. 2017) and those that assume other fixed limits (e.g., divisive normalization-based limits; Bays, 2015). In contrast, models of visual working memory that argue that all that is being assessed, even in continuous report paradigms, is the signal-to-noise ratio of the memory trace (d'; Schurgin et al. 2020) take a much more fluid approach to the concept of fixed capacity. Schurgin et al. (2020), for example, point out that it would not be surprising for memories to become noisier when more items need to be encoded, or to become noisier as delay increases, and that combining multiple sources of change in signal strength (e.g., splitting attention at encoding; changing encoding time) with multiple sources of change in noise accumulation (e.g., splitting maintenance capacity; increased delay) is unlikely to result in a single fixed capacity being observed in memory performance, even if there is, deep down, some underlying limited resource in some of the components that affect signal and noise.

\section{Contribution of 'long'-term memory}

Many studies have shown that existing knowledge or familiarity with a stimulus improves the ability to maintain information not only in long-term memory, but also over short delays, often termed long-term working memory (Ericsson \& Kintsch, 1995). However, these other forms of working memory, which are thought to be, possibly, non-active, are sometimes not considered to be core elements of working memory capacity (Awh \& Vogel, 2020), even though they play major roles in most cognitive theories of working memory and are likely critical to performing everyday tasks (e.g., Cowan, 2005; Ericsson \& Kintsch, 1995). Instead, the active component of working memory (sometimes referred to as the 'focus of attention') is often considered particularly important (e.g., Cowan, 2005). One common question is thus the extent to which real-world object benefits arise from changes in active storage in working memory per se; or from the usage of 'long-term' memory systems or other forms of more passive storage that can be utilized in the short-term maintenance of information, like 'activated long-term memory'; or even the extent to which these concepts are truly dissociable.

To address this issue in the present study, Experiment 2 was designed to indirectly estimate the role of more durable storage - including activated long-term memory and other more persistent short-term storage that is sometimes found to play a role in short-term storage of real-world objects (Endress \& Potter, 2014; Makovski, 2016). In particular, this experiment allowed us to examine the role of spatial cues for colors and real-world objects, which past research has indicated is a dominant factor in determining the relative contribution of active storage in 
working memory vs. the formation of more durable traces. In particular, when items are presented at the same location sequentially, research has shown that people rely on more durable memory traces as suggested by the presence of more proactive interference in these task conditions; whereas when items are presented at spatially distinct locations, there appears to be little lingering information trial-to-trial (Makovski, 2016). This finding is consistent with models that suggest that active storage in working memory makes use of sustaining activity in the visual system (e.g., Serences, 2016): insofar as much of visual cortex, including higher-level cortex, is retinotopically or at least spatially organized (e.g., Golomb \& Kanwisher, 2012), actively maintaining information may make binding to spatial location straightforward. Eliminating such spatial cues thus may decrease the tendency for participants to actively hold items in mind and cause them to rely on more durable working memory traces, like activated long-term memory traces.

One strong prediction you would make based on this previous work is that if more durable memory traces are particularly relevant to performance with real-world objects, but not colors, then most object benefits should occur when all objects are presented at the same location in the center of the screen, and such benefits should be relatively small or non-existent when items are spatially presented and spatially cued. However, we found large and comparable benefits in both cases: regardless of whether items were presented at the same or different locations, meaningful objects were much better remembered than colors (Exp. 2). This provides evidence that the present effects are not straightforwardly explained by differences in the durability of memory and thus different memory systems being used for objects vs. colors.

Another general concern when measuring working memory for meaningful stimuli is the contribution of not only these different more durable memory systems, but also the possibility that participants may take advantage of long encoding times to re-code stimuli verbally - as verbal encoding would clearly result in fundamentally different memory traces. In the current work, to reduce the possibility of participants relying on verbal encoding, we had them perform a concurrent articulatory suppression task to hinder them from using verbal labels, and we actively monitored performance on this task in Experiments 1 and 3 . Notably, the effects in the experiments with the verbal interference task actively monitored by experimenters and the replications without active monitoring of performance of this task - and thus where participants may be less inclined to perform it continuously - are similar, suggesting that verbal encoding and rehearsal strategies may not play a significant role in any of these studies. 


\section{Active neural representation}

The present data are consistent with the results obtained in previous studies that used neural measures to assess how much information was actively held in working memory. In particular, the contralateral delay activity (CDA) is an EEG marker that has been associated with how much information is actively held in mind (Vogel \& Machizawa, 2004). Brady et al. (2016) found that real-world objects resulted in higher memory performance than simple colors, and this performance increase was accompanied by an increase in the CDA component, suggesting it was supported by active storage in working memory. Similarly, Asp, Störmer, \& Brady (2019) showed that when participants remembered ambiguous Mooney face stimuli, memory performance and CDA were increased when participants recognized the stimuli as meaningful (i.e., a face) relative to when they just saw them as meaningless black and white shapes (see also Xie \& Zhang, 2017). Critically, by asking participants whether they perceived the stimuli as a face or not, that study also showed that enhancements in active maintenance of items in visual working memory are due to the subjective perception of the stimulus as meaningful, and are not driven by physical properties of the stimulus. Evidence from fMRI is also consistent with these results: for example, Veldsman et al. (2017) found evidence of richer representations in critical working memory regions in parietal cortex for meaningful rather than perceptuallymatched non-meaningful stimuli; and Stojanoski et al. (2019) found evidence that meaningful stimuli were processed in more high-level ventral regions in preparation for visual working memory storage than perceptually-matched non-meaningful stimuli.

In contrast to this significant literature, one paper has claimed - without actually finding behavioral object benefits - that such benefits may derive from non-active storage (Quirk et al. 2020). It is unclear what the origin of the difference between Quirk et al. (2020) and Brady et al. (2016), Asp et al. (2019), Stojanoski et al. (2019), Starr et al. (2020) and others is, although the current work suggests some possibilities, including the usage of more holistic encoding strategies in their participants and non-matched target/foil similarity across their stimulus sets (see Brady \& Störmer, 2020).

While they have taken on an outsize importance in this particular subfield, it is also not clear that thinking solely about neural measures like the CDA, rather than thinking about the cognitive operations people actually do to perform tasks (as in the origin of the term 'working memory'), is actually of critical relevance when considering working memory capacity limits and how they differ for different stimuli. In theory, neither behavioral evidence based on the role of spatial 
locations in proactive interference nor neural markers of working memory storage like the CDA provide definitive proof that items are held actively in mind in working memory. Interpretations of the CDA, for example, are open to issues of reverse inference and circularity - the CDA is claimed to measure working memory because it tracks behavioral performance in some circumstances (e.g., Vogel \& Machizawa, 2004). Then, if the CDA does not track behavior in a given instance, is this because people are not using working memory for all the information (e.g., Quirk et al. 2020), or because the CDA does not provide a pure index of working memory, but instead something more like attention (e.g., Berggren, \& Eimer, 2016), or does not index active maintenance memory signals that are known to be present but are not purely lateralized (Robitaille et al. 2010)? Clearly, such issues cannot be settled straightforwardly. Nevertheless, we think the evidence favors a view where behavioral benefits for meaningful stimuli are interpreted straightforwardly: People are better able to remember objects and other meaningful stimuli over short delays; in many circumstances, this is tracked by increased neural activity consistent with working memory usage; there is evidence that stimulus-specific brain regions are engaged for working memory for meaningful stimuli alone (e.g., Druzgal \& D'Esposito, 2001; Galvez-Pol et al. 2018); and such effects seem relatively unaffected by verbal encoding or diversity in spatial locations that affect proactive interference. This is altogether indicative of enhanced active storage in working memory for meaningful stimuli.

\section{Why real-world objects have a higher working memory capacity}

In the current work, we investigated what conditions lead to the strongest benefits for meaningful stimuli. We found large advantages for meaningful objects in all conditions, but also found that real-world objects - and to a lesser degree lightly scrambled versions of the objects - benefit from the sequential encoding and thus deeper, focused-on-individual-items processing, while colors and non-meaningful fully scrambled objects do not. Our results suggest meaningless, and particularly single feature objects, may be an outlier in their affordance of parallel, quick processing, and that in more realistic memory situations, visual working memory likely relies upon representations resulting from in-depth processing of objects rather than solely being represented in terms of their low-level features. In particular, people may maintain information in visual working memory not solely in terms of colors and shapes and other 'basic' visual dimensions in low-level visual cortex (e.g., Serences, 2016), but also maintain active representations of the stimuli in higher-level visual regions (e.g., fusiform face area (FFA) for face stimuli: Druzgal \& D'Esposito, 2001; somatosensory regions for hand images: Galvez-Pol et al. 2018), resulting in stronger memories for these items (e.g., Stojanoski et al. 2019; Asp, 
Störmer \& Brady, 2019; Brady et al., 2016). This may not only provide more potential sites of storage, but may also limit interference between the neural populations that must be held active, producing more distinct memories for different objects (e.g., Cohen et al. 2014; Wyble et al. 2016). Overall, then, we suggest that the working memory system can capitalize on knowledge — and connections to knowledge, enhanced by deeper processing — thereby building stronger and more robust memory representations for meaningful stimuli.

\section{Acknowledgements}

This work was supported by an NSF grant (BCS-1829434) to TFB and VSS. We thank Kevin Sayed for help with data collection.

\section{References}

Adam, K. C., Vogel, E. K., \& Awh, E. (2017). Clear evidence for item limits in visual working memory. Cognitive psychology, 97, 79-97.

Alvarez, G. A., \& Cavanagh, P. (2004). The capacity of visual short-term memory is set both by visual information load and by number of objects. Psychological science, 15(2), 106-111.

Asp, I., Störmer, V. S., \& Brady, T. (2019). Greater visual working memory capacity for visuallymatched stimuli when they are recognized as meaningful. PsyArxiv, https://doi.org/10.31234/osf.io/r6njf

Awh, E., Barton, B., \& Vogel, E. K. (2007). Visual working memory represents a fixed number of items regardless of complexity. Psychological science, 18(7), 622-628.

Awh, E. \& Vogel, E.K, (2020) Online and Off-Line Memory States in the Human Brain. In the Cognitive Neurosciences, edited by Poeppel, Mangun \& Gazzaniga.

Babic, Z., Schurgin, M.W., and Brady, T.F. (2019). Is short-term storage correlated with fluid intelligence? Strategy use explains the apparent relationship between 'number of remembered items' and fluid intelligence. PsyArXiv, https://doi.org/10.31234/osf.io/83ch4

Baddeley, A. (2012). Working memory: Theories, models, and controversies. Annual review of psychology, 63, 1-29. 
Ballard, D. H., Hayhoe, M. M., \& Pelz, J. B. (1995). Memory representations in natural tasks. Journal of cognitive neuroscience, $7(1), 66-80$.

Bays, P. M., Catalao, R. F., \& Husain, M. (2009). The precision of visual working memory is set by allocation of a shared resource. Journal of vision, 9(10), 7.

Bays, P. M., Gorgoraptis, N., Wee, N., Marshall, L., \& Husain, M. (2011). Temporal dynamics of encoding, storage, and reallocation of visual working memory. Journal of vision, 11(10), 6-6.

Bays, P. M. (2015). Spikes not slots: noise in neural populations limits working memory. Trends in cognitive sciences, 19(8), 431-438.

Berggren, N., \& Eimer, M. (2016). Does contralateral delay activity reflect working memory storage or the current focus of spatial attention within visual working memory?. Journal of Cognitive Neuroscience, 28(12), 2003-2020.

Brady, T. F., Konkle, T., Alvarez, G. A. and Oliva, A. (2008). Visual long-term memory has a massive storage capacity for object details. Proceedings of the National Academy of Sciences, 105 (38), 14325-14329.

Brady, T. F. and Alvarez, G.A. (2015a). Contextual effects in visual working memory reveal hierarchically structured memory representations. Journal of Vision, 15(15):6

Brady, T. F., \& Alvarez, G. A. (2015b). No evidence for a fixed object limit in working memory: Spatial ensemble representations inflate estimates of working memory capacity for complex objects. Journal of Experimental Psychology: Learning, Memory, and Cognition, 41(3), 921.

Brady, T.F. \& Störmer, V.S (2020). Greater capacity for objects than colors in visual working memory: Comparing memory across stimulus spaces requires maximally dissimilar foils. PsyArxiv.

Brady, T. F., Konkle, T., \& Alvarez, G. A. (2009). Compression in visual working memory: Using statistical regularities to form more efficient memory representations. Journal of Experimental Psychology: General, 138(4), 487.

Brady, T. F., Störmer, V. S., \& Alvarez, G. A. (2016). Working memory is not fixed-capacity: More active storage capacity for real-world objects than for simple stimuli. Proceedings of the National Academy of Sciences, 113(27), 7459-7464.

Cohen, M. A., Konkle, T., Rhee, J. Y., Nakayama, K., \& Alvarez, G. A. (2014). Processing multiple visual objects is limited by overlap in neural channels. Proceedings of the National Academy of Sciences, 111(24), 8955-8960. 
Cowan, N. (2001). The magical number 4 in short-term memory: A reconsideration of mental storage capacity. Behavioral and brain sciences, 24(1), 87-114.

Cowan N. (2005) Working memory capacity. Hove, East Sussex, UK: Psychology Press.

Curby, K. M., Glazek, K., \& Gauthier, I. (2009). A visual short-term memory advantage for objects of expertise. Journal of Experimental Psychology: Human Perception and Performance, 35(1), 94.

Cusack, R., Lehmann, M., Veldsman, M., \& Mitchell, D. J. (2009). Encoding strategy and not visual working memory capacity correlates with intelligence. Psychonomic bulletin \& review, 16(4), 641-647.

Druzgal, T. J., \& D'Esposito, M. (2001). Activity in fusiform face area modulated as a function of working memory load. Cognitive Brain Research, 10(3), 355-364.

Endress, A. D., \& Potter, M. C. (2014). Large capacity temporary visual memory. Journal of Experimental Psychology: General, 143(2), 548.

Ericsson, K. A., \& Kintsch, W. (1995). Long-term working memory. Psychological Review, 102(2), 211-245. https://doi.org/http://dx.doi.org/10.1037/0033-295X.102.2.211

Galvez-Pol, A., Calvo-Merino, B., Capilla, A., \& Forster, B. (2018). Persistent recruitment of somatosensory cortex during active maintenance of hand images in working memory. Neuroimage, 174, 153-163.

Golomb, J. D., \& Kanwisher, N. (2012). Higher level visual cortex represents retinotopic, not spatiotopic, object location. Cerebral Cortex, 22(12), 2794-2810.

Hayhoe, M. M., Shrivastava, A., Mruczek, R., \& Pelz, J. B. (2003). Visual memory and motor planning in a natural task. Journal of vision, 3(1), 6-6.

Hollingworth, A., Richard, A. M., \& Luck, S. J. (2008). Understanding the function of visual shortterm memory: transsaccadic memory, object correspondence, and gaze correction. Journal of Experimental Psychology: General, 137(1), 163.

Jackson, M. C., \& Raymond, J. E. (2008). Familiarity enhances visual working memory for faces. Journal of Experimental Psychology: Human Perception and Performance, 34(3), 556. 
Li, X., Xiong, Z., Theeuwes, J., \& Wang, B. (2020). Visual memory benefits from prolonged encoding time regardless of stimulus type. Journal of Experimental psychology. Learning, Memory, and Cognition.

Lin, P. H., \& Luck, S. J. (2012). Proactive interference does not meaningfully distort visual working memory capacity estimates in the canonical change detection task. Frontiers in psychology, 3, 42.

Linke, A. C., Vicente-Grabovetsky, A., Mitchell, D. J., \& Cusack, R. (2011). Encoding strategy accounts for individual differences in change detection measures of VSTM. Neuropsychologia, 49(6), 1476-1486.

Luck, S. J., \& Vogel, E. K. (1997). The capacity of visual working memory for features and conjunctions. Nature, 390(6657), 279-281.

Makovski, T. (2016). Does proactive interference play a significant role in visual working memory tasks?. Journal of Experimental Psychology: Learning, Memory, and Cognition, 42(10), 1664.

Nassar, M. R., Helmers, J. C., \& Frank, M. J. (2018). Chunking as a rational strategy for lossy data compression in visual working memory. Psychological review, 125(4), 486.

Ngiam, W. X., Khaw, K. L., Holcombe, A. O., \& Goodbourn, P. T. (2019). Visual working memory for letters varies with familiarity but not complexity. Journal of Experimental Psychology: Learning, Memory, and Cognition, 45(10), 1761.

O'Donnell, R. E., Clement, A., \& Brockmole, J. R. (2018). Semantic and functional relationships among objects increase the capacity of visual working memory. Journal of Experimental Psychology: Learning, Memory, and Cognition, 44(7), 1151.

Quirk, C., Adam, K.C.S., \& Vogel, E.K. (2020). No evidence for an object working memory capacity benefit with extended viewing time. PsyArxiv, https://osf.io/pzy5q/

Robitaille, N., Marois, R., Todd, J., Grimault, S., Cheyne, D., \& Jolicœur, P. (2010).

Distinguishing between lateralized and nonlateralized brain activity associated with visual shortterm memory: fMRI, MEG, and EEG evidence from the same observers. Neurolmage, 53(4), 1334-1345.

Rousselet, G. A., Thorpe, S. J., \& Fabre-Thorpe, M. (2004). How parallel is visual processing in the ventral pathway?. Trends in cognitive sciences, 8(8), 363-370. 
Sahar, T., Sidi, Y., \& Makovski, T. (2020). A Metacognitive Perspective of Visual Working Memory With Rich Complex Objects. Frontiers in Psychology, 11, 179.

Salahub, C., Lockhart, H. A., Dube, B., Al-Aidroos, N., \& Emrich, S. M. (2019).

Electrophysiological correlates of the flexible allocation of visual working memory resources. Scientific Reports, 9(1), 1-11.

Salmela, V. R., Ölander, K., Muukkonen, I., \& Bays, P. M. (2019). Recall of facial expressions and simple orientations reveals competition for resources at multiple levels of the visual hierarchy. Journal of vision, 19(3), 8-8.

Schurgin, M. W., Wixted, J. T., \& Brady, T. F. (2018). Psychophysical scaling reveals a unified theory of visual memory strength. BioRxiv, 325472.

Serences, J. T. (2016). Neural mechanisms of information storage in visual short-term memory. Vision research, 128, 53-67.

Shoval, R., \& Makovski, T. (2019). The problem of meaning: Meaningful stimuli alter visual working memory performance. Paper presented at Object Perception, Attention and Memory (OPAM), Montreal, Canada.

Starr, A. Srinivasan, M., Bunge, S. (2020). Semantic knowledge influences visual working memory in children and adults. PLoS One.

Stojanoski, B., Emrich, S. M., \& Cusack, R. (2019). Representation of semantic information in ventral areas during encoding is associated with improved visual short-term memory. bioRxiv.

Suchow, J. W., Brady, T. F., Fougnie, D. and Alvarez, G. A. (2013). Modeling visual working memory with the MemToolbox. Journal of Vision, 13(1), 9.

Treisman, A. M., \& Gelade, G. (1980). A feature-integration theory of attention. Cognitive psychology, 12(1), 97-136.

Tsubomi, H., Fukuda, K., Watanabe, K., \& Vogel, E. K. (2013). Neural limits to representing objects still within view. Journal of Neuroscience, 33(19), 8257-8263.

Veldsman, M., Mitchell, D. J., \& Cusack, R. (2017). The neural basis of precise visual short-term memory for complex recognisable objects. Neurolmage, 159, 131-145.

Vogel, E. K., \& Machizawa, M. G. (2004). Neural activity predicts individual differences in visual working memory capacity. Nature, 428(6984), 748-751. 
Vogel, E. K., Woodman, G. F., \& Luck, S. J. (2006). The time course of consolidation in visual working memory. Journal of Experimental Psychology: Human Perception and Performance, 32(6), 1436.

White, A. L., Runeson, E., Palmer, J., Ernst, Z. R., \& Boynton, G. M. (2017). Evidence for unlimited capacity processing of simple features in visual cortex. Journal of vision, 17(6), 19-19.

Whitney, D., \& Levi, D. M. (2011). Visual crowding: A fundamental limit on conscious perception and object recognition. Trends in cognitive sciences, 15(4), 160-168.

Wyble, B., Swan, G., \& Callahan-Flintoft, C. (2016). Measuring Visual Memory in Its Native Format. Trends in Cognitive Sciences, 20(11), 790-791

Xie, W. \& Zhang, W. (2017). Familiarity increases the number of retained Pokémon in visual short-term memory. Memory \& Cognition. 45(4), 677-689

Zhang, W., \& Luck, S. J. (2008). Discrete fixed-resolution representations in visual working memory. Nature, 453(7192), 233-235.

\section{Appendix: Scrambled stimuli validation}

To examine the relative amount of meaning that was preserved by our lightly scrambled and fully scrambled images, we ran a separate stimulus validation study where we asked participants to recognize the images. As previous work has clearly indicated the fully scrambled images cannot be recognized per se (i.e., participants cannot spontaneously tell that they are; Stojanoski \& Cusack, 2014), we attempted to make a task where at least some inferences about what object it might be could be extracted even from these fully scrambled images, to allow a continuous measure of how much meaning could be extracted from the objects. In particular, we showed one image - which could be an original object image, a lightly scrambled object or a fully scrambled object - along with two verbal labels, one of which applied to the image and one of which was instead from a different object. We asked them to, as quickly as possible, indicate which label was appropriate. We reasoned that by examining both accuracy and reaction time, we can see how easily the images are recognized and how much semantic information is preserved. And, because the fully scrambled images preserve color and topology, we expected that at least some relevant information could be extracted about them in this task, allowing us to compare their overall difficulty to that of the lightly scrambled and intact objects. 


\section{Method}

Participants. 30 participants were recruited via Prolific. All were age 18-35, had normal or corrected-to-normal vision, and were in the United States. One participant was excluded posthoc because they were below chance at picking the appropriate label in the regular object condition, leaving 29 participants.

Procedure. On each trial, participants saw an image and two possible verbal labels (to the left and right of the image). They then indicated as quickly and accurately as possible which label was appropriate for the image using the ' $z$ ' and ' $m$ ' keys (for left and right, respectively). Each participant did 120 trials: 40 were original objects; 40 were lightly scrambled; and 40 were fully scrambled.

We chose the 40 objects per condition as follows: In our memory experiments, we had 240 pairs of objects that served as the tested items in each study (i.e. in the memory studies, the tested study item and foil were from a given pair). In this stimulus validation experiment, we used only 240 total object images, selecting just 1 from each pair. For each participant, we then took these 240 images, and used half of them as the images that participants would be probed on; and the other 120 gave the foil labels that would be presented on each trial. This ensured the foil labels were not ones that were associated with images that were actually seen in the experiment. The 120 objects that were presented to participants, were then divided equally into each of the 3 conditions, so that the same objects would not be presented as scrambled vs. intact.

\section{Results}

We found a main effect of scrambling level on accuracy, $F(2,56)=95.13, p<0.0001$. Intact objects were more accurately recognized than lightly scrambled objects $(t(28)=3.13, p=0.004)$ and both were more accurately recognized than fully scrambled objects ( $p s<0.0001)$. Similarly, in terms of median reaction time per participant per condition, there was a main effect of scrambling $(F(2,56)=120.86, p<0.0001)$, and all pairwise comparisons were significant (ps $<0.0001)$. 

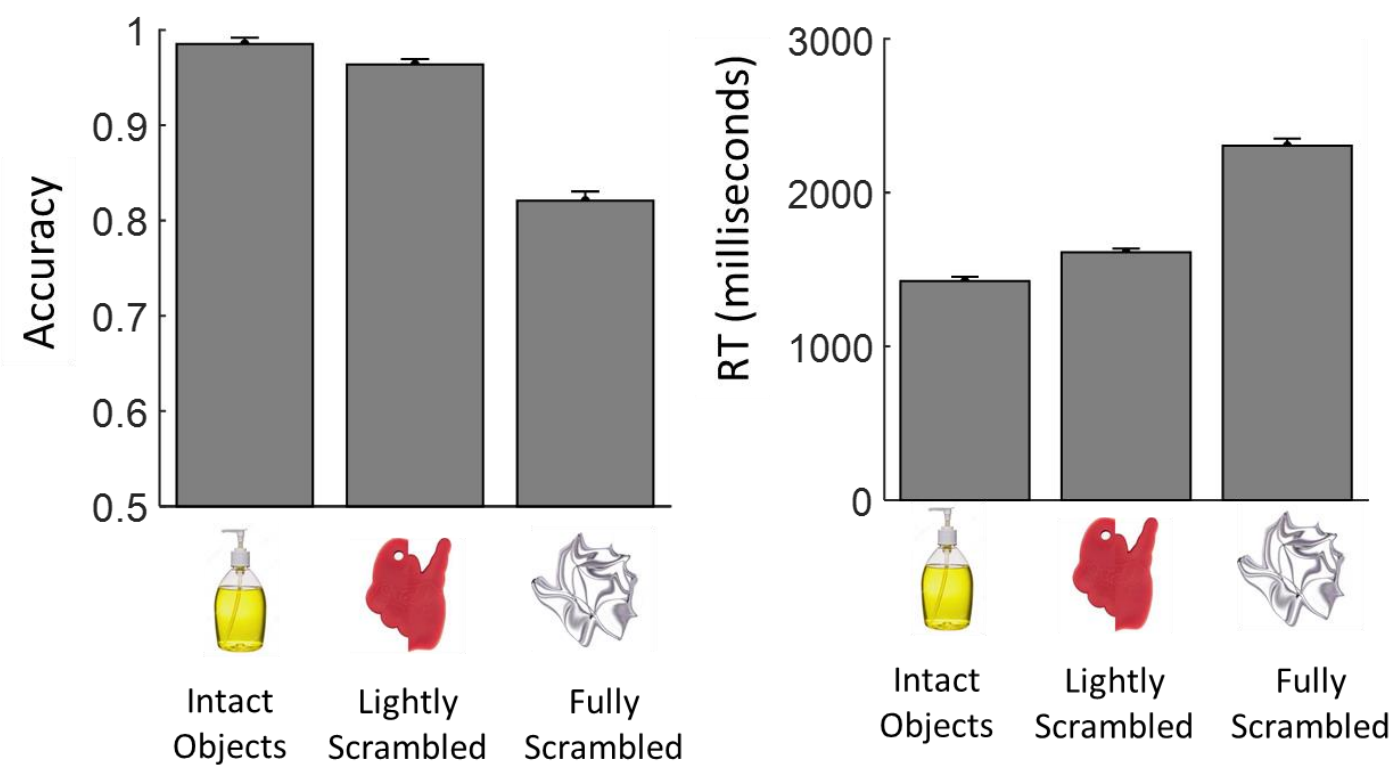

Figure A1. Both accuracy and speed of matching an object with the appropriate label were strongly impacted by scrambling, with the fully scrambled objects impaired to a much greater extent than the lightly scrambled objects.

Overall, the data show that the correct label for intact objects was quickly and accurately recognized. By comparison, lightly scrambled objects were recognized slightly more slowly and slightly less accurately. However, responses for fully scrambled objects were quite inaccurate and quite slow, even with only two choices, and even though the full scramble preserves color and topography as well as most low-level features.

In general, these data suggest that lightly scrambled objects were much closer to intact objects than to fully scrambled objects, validating the idea that they retain significant amounts of meaningful information. Previous work has shown the fully scrambled objects retain little in the way of semantic information (Stojanoski \& Cusack, 2014) and our results are consistent with that - even with an extremely straightforward task of picking between two labels, where in many cases color alone may be sufficient, participants were quite inaccurate and quite slow with these fully scrambled images. 